\title{
Manipulating the Optical Bistability in a Nonlinear Plasmonic Nanoantenna Array with a Reflecting Surface
}

\author{
Jérémy Butet • Olivier J. F. Martin
}

Received: 30 June 2014 / Accepted: 1 September 2014 / Published online: 12 September 2014

(C) Springer Science+Business Media New York 2014

\begin{abstract}
The influence of a reflecting surface on the optical bistability in a nanoantenna array is investigated theoretically. The optical response of the array is modeled using a surface integral equation method developed for periodic structures, and the description of the Kerr effect is based on an analytical model. Different behaviors are observed when the distance between the nanoantenna array and the silver layer is changed. Indeed, a modification of the nanoantennas radiative properties permit to control important parameters of the nonlinear response such as the intensity threshold and the area of the hysteresis cycle. The results presented in this article demonstrate that a reflecting surface is a convenient and flexible tool for controlling the operating of nonlinear optical systems based on the Kerr effect.
\end{abstract}

Keywords Optical bistability · Nanoantenna $\cdot$ Kerr effect . Nonlinear plasmonics $\cdot$ Surface integral equation method

\section{Introduction}

Metallic nanostructures support surface plasmon resonances corresponding to the collective excitations of the conduction electrons and exhibit unique optical properties [1]. The coupling between several substructures is a convenient way to control surface plasmon resonance properties [2-4]. In particular, bringing two nanostructures in close proximity results in an enhancement by several orders of magnitude for the electric field in the nanogap, producing a so-called hot spot. This geometry is called a nanoantenna and represents the optical

\section{J. Butet $(\bowtie) \cdot$ O. J. F. Martin}

Nanophotonics and Metrology Laboratory (NAM), Swiss Federal

Institute of Technology Lausanne (EPFL), Lausanne 1015,

Switzerland

e-mail: jeremy.butet@epfl.ch analogous of microwave and radiowave antennas [5-7]. The electromagnetic properties of nanoantennas can be tuned by modifying their geometric parameters (length, shape, and gap dimension) and tailored for specific applications [8]. For instance, optical antennas have been designed for studying quantum systems at the single emitter level [9-11], surfaceenhanced Raman scattering [12, 13], and trapping [14-16]. It was shown that the radiative properties of nanoantennas can be controlled using a reflecting metallic surface $[17,18]$. For example, this feature has been previously used to enhance the fluorescence and surface-enhanced Raman scattering for molecules close to metallic nanoantennas [19,20].

Optical antennas are also promising for the observation of nonlinear optical effects which require the high electric field observed in the hot spots [21]. Several studies have discussed multi-photon-excited luminescence [22], second harmonic generation [23-26], third harmonic generation [27, 28], and four-wave mixing $[29,30]$. The nonlinear Kerr effect in plasmonic nanostructures coupled to nonlinear materials has also been investigated in several publications [31-35]. Including a Kerr material in plasmonic nanostructures is a convenient way to achieve the control of light by light at the nanoscale and optical memories, transistors, and switches can be designed using this approach [36, 37].

In this article, a new approach to control the Kerr effect down to the nanoscale is introduced. A reflecting surface is used to modify the coupling between the incident light and a nanoantenna array, and the influence on its nonlinear optical response is investigated. The optical response of the array is modeled using a surface integral equation (SIE) method involving a periodic Green's function. The Kerr effect is described using an analytical model which was previously introduced for isolated nanoantennas loaded with a nonlinear material [33] and modified here for the description of nanoantenna arrays close to a metallic surface. In particular, a silver layer is added below the dielectric layer, and the influence of 
the distance between the metallic surface and the nanoantenna array on the nonlinear response is studied in details. The results presented in this article demonstrate that a reflecting surface is a convenient way of controlling the operation of nonlinear optical systems.

\section{Results and Discussion}

The article is organized as follows. First, the linear optical response of a nanoantenna array on a $\mathrm{SiO}_{2}$ substrate is evaluated using a SIE method [38, 39]. The influence of a reflective silver surface on this response is then discussed in details. Finally, the nonlinear response resulting from the Kerr material loaded in the nanoantennas gaps is investigated using an analytical model adapted from [33].

\section{Linear Response of Nanoantennas Array}

Let us first consider the linear optical response of a nanoantenna array. The nanoantenna arms are made of silver, and the nanogaps are completely filled with polystyrene (linear refractive index $n_{0}=1.60$ ). The length, width, and height of the rectangular arms are 60,20 , and $20 \mathrm{~nm}$, respectively. The arms are separated by a $10-\mathrm{nm}$ nanogap. The period of the two-dimensional array is $500 \mathrm{~nm}$ in both directions. All these parameters are kept constant in the following discussion. The nanoantenna array is deposited on a semi-infinite $\mathrm{SiO}_{2}$ substrate (refractive index $n=1.55$ ). The linear electromagnetic response of this system is computed with the SIE method using a periodic Green's function [38, 39]. This method requires the discretization of the structure in the unit cell only and was found to be very accurate even in resonant conditions $[38,39]$. The surface of the plasmonic nanoantenna is discretized with a triangular mesh with a typical side of $5 \mathrm{~nm}$, and the surface of the substrate is discretized with a triangular mesh with a typical side of $25 \mathrm{~nm}$. Indeed, a finer mesh is required to reproduce the significant electromagnetic field variation close to plasmonic nanostructures and nanogaps [40]. The array is excited by a plane wave polarized along the nanoantenna arms and propagating along the normal to the $\mathrm{SiO}_{2}$ surface. The reflectance of the nanoantenna array is shown as a function of the incident wavelength in Fig. 1b. The dielectric constant of silver is taken from experimental data [41]. A strong increase of the reflectance is observed as the incident wavelength is tuned close to $710 \mathrm{~nm}$, corresponding to the resonant wavelength of the surface plasmon mode supported by the nanoantennas. In the next section, a reflecting silver surface is placed below a finite thickness $\mathrm{SiO}_{2}$ substrate, and its influence on the optical response of the nanoantenna array is investigated.
Influence of the Reflecting Surface

In order to understand the role of a reflective surface, we first consider $\mathrm{a} \mathrm{SiO}_{2}$ layer without nanoantenna array deposited on a semi-infinite silver substrate (Fig. 2a). When the incident and the reflected waves interfered constructively at the molecule position, the optical intensity is increased. This condition can be satisfied by modifying the dielectric spacer, i.e., the $\mathrm{SiO}_{2}$ layer thickness. Considering the reflection at the interface of a perfect electric conductor, the optimal thickness corresponding to constructive interference is given by [18]:

$2 d n=\left(m+\frac{1}{2}\right) \lambda$

where $n$ is the refractive index of the dielectric spacer $\left(\mathrm{SiO}_{2}\right.$ in the present case), $d$ is the dielectric spacer thickness, $\lambda$ is the incident wavelength in vacuum, and $m$ is an integer. Considering an incident wavelength corresponding to surface plasmon resonance supported by the nanoantenna array discussed previously $(\lambda=710 \mathrm{~nm})$, the first thickness leading to constructive interference is found to be $d=114 \mathrm{~nm}$. To confirm this, the electric field intensity is evaluated $10 \mathrm{~nm}$ above the $\mathrm{SiO}_{2}$ layer as a function of its thickness using the SIE method. An intensity maximum is observed for a dielectric layer thickness between 80 and $100 \mathrm{~nm}$. This thickness is slightly shorter than the value predicted by the Eq. (1) since the incoming light penetrates into the silver slab, which is not a perfect conductor at this wavelength, resulting in an additional phase shift [19].

We now include the nanoantenna array on the dielectric substrate and investigate the influence of the silver surface on its optical response. The system is then composed of a nanoantenna array, a $\mathrm{SiO}_{2}$ dielectric spacer, and a semi-infinite silver slab (Fig. 3a). The reflectance is computed for thicknesses $d$ between 100 and $200 \mathrm{~nm}$. The result is shown as a function of the incident wavelength in Fig. 3b. The different reflectance spectra were fitted with the function:

$R=1-\frac{2 A_{0}}{\pi} \frac{w}{4\left(\lambda-\lambda_{c}\right)^{2}+w^{2}}$,

where $\lambda$ is the incident wavelength, $\lambda_{\mathrm{c}}$ is the dip wavelength, $w$ is the full width at half minimum, and $\mathrm{A}_{0}$ is a fitting parameter, with units of a length, related to the dip magnitude. The fits are shown as continuous lines in Fig. 3b. It is found that the reflectance dramatically depends on the dielectric spacer thickness. For the largest studied thickness $(d=200 \mathrm{~nm})$, the reflectance does not depend on the incident wavelength and is close to unity. This behavior is easily understood from Fig. $2 b$, which indicates that the intensity vanishes at the nanoantenna position in this configuration. As a consequence, the surface plasmon resonance supported by the nanoantennas is not excited and does not modify the optical response of the system, leading to a 


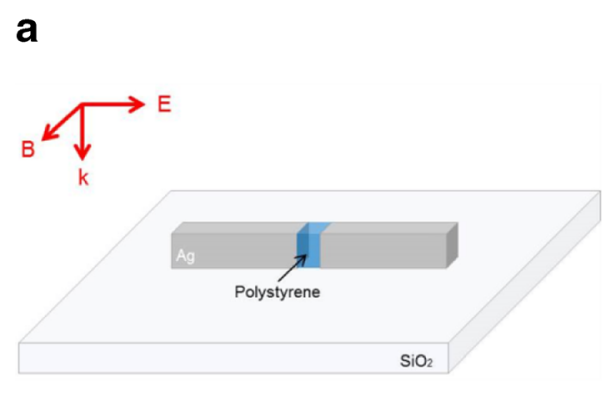

Fig. 1 a Schematic of a silver dipolar nanoantenna loaded with polystyrene and deposited on $\mathrm{SiO}_{2}$ substrate. $\mathbf{b}$ Reflectance of a periodic array of silver nanoantennas loaded with polystyrene on a $\mathrm{SiO}_{2}$ substrate as a function of the incident wavelength. The spatial period is $500 \mathrm{~nm}$. The

flat response for $d=200 \mathrm{~nm}$ in Fig. 3b. This behavior has already been discussed in the case of a single plasmonic nanorod inside a microcavity [42]. According to Fig. 2b, the coupling between the electromagnetic wave and the periodic array of silver nanoantennas must increase when the thickness $d$ decreases. In this case, the interference between the incoming and the reflected waves are not totally destructive at the nanoantenna position. The excitation of the surface plasmon resonance supported by the nanoantennas results in the formation of a dip in the reflectance spectra (Fig. 3b). As the coupling increases, i.e., as the distance decreases, this dip becomes deeper and broader, revealing that the radiative behavior of the system is indeed modified. The maximum reflectance dip corresponds to the maximum field enhancement in the nanoantenna gaps, as reported in [18]. We will show that this modification of the intensity enhancement in the nanoantenna gap can be used to control the nonlinear response of the nanoantenna array.

\section{Nonlinear Kerr Effect}

In this section, the nonlinear Kerr effect in the polystyrene part loaded in the nanoantenna gaps is taken into account.

Fig. 2 a Schematic of a $\mathrm{SiO}_{2}$ film on a silver substrate. $\mathbf{b}$ Intensity as a function of $\mathrm{SiO}_{2}$ thickness $d$ evaluated $10 \mathrm{~nm}$ above the $\mathrm{SiO}_{2}$ substrate (in air). The wavelength of the incident plane wave is $\lambda=$ $710 \mathrm{~nm}$

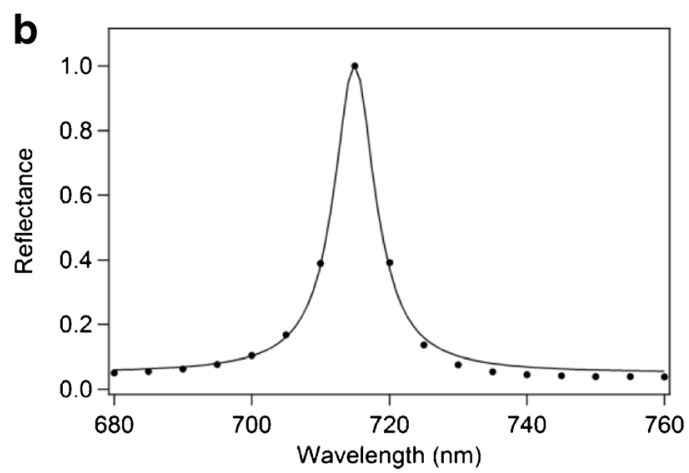

incident plane wave is polarized along the nanoantenna arms and its wave vector is directed along the normal to the $\mathrm{SiO}_{2}$ surface. The incident wave is incident from the air side. The continuous line is a fit with a Lorentzian function

Reference [33] introduces an analytical model to describe the Kerr effect in isolated plasmonic nanoantennas. This model assumes that the electric field distribution is uniform in the nanogap, resulting in a uniform refractive index change. Fig $3 \mathrm{a}$ indicates that this approximation is also valid in the present case, where the intensity in the nanogap does not vary by more than a third of the maximum intensity in the nanogap center (Fig. 3a). This previous analytical model is now modified to describe the case of a nanoantenna array close to a reflecting surface with the aim to determine the relation between the incident intensity and the reflectance. To describe the Kerr nonlinearity, the refractive index of the polystyrene is now a function of the intensity [43]:

$n=n_{0}+n_{2} I$

where $n_{0}$ is the linear part of the refractive index, $n_{2}$ is the nonlinear Kerr coefficient, and $I$ is the intensity in the Kerr material, i.e., in the nanogap. The intensity $I$ is the intensity of the electric field and $I=1 / 2 \varepsilon_{0}|\mathrm{E}|^{2}$. The nonlinear Kerr coefficient of polystyrene is $n_{2}=1.14 \times 10^{-12} \mathrm{~cm}^{2} / \mathrm{W}$ [44]. This coefficient is positive and the Kerr effect leads to an increase of the refractive index of polystyrene. In order to determine the
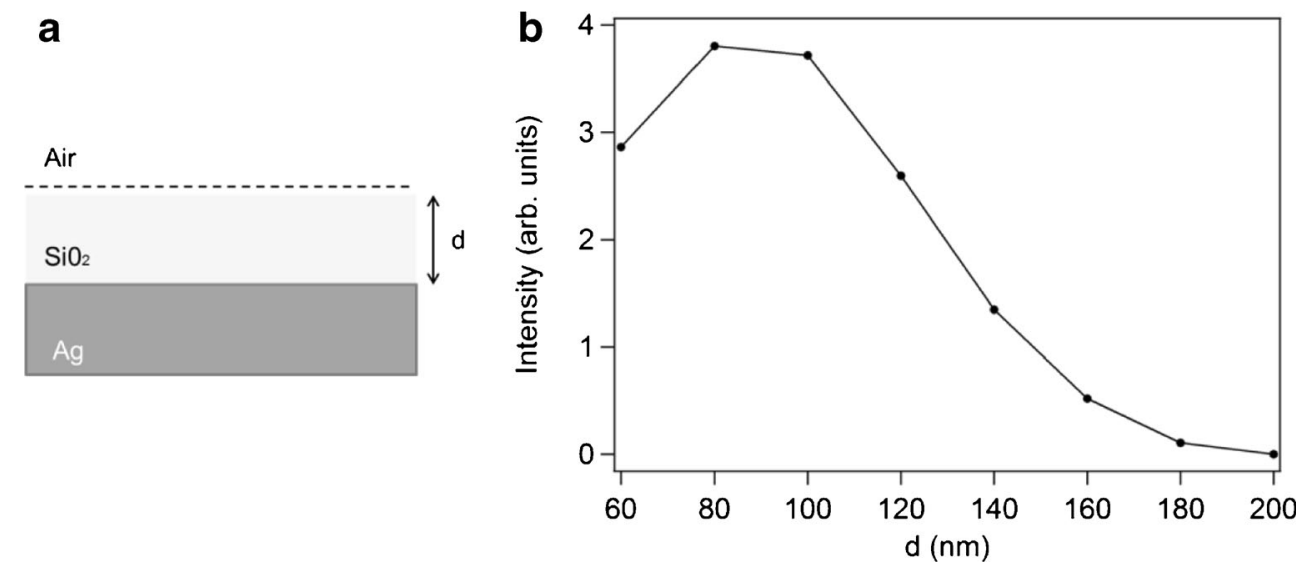

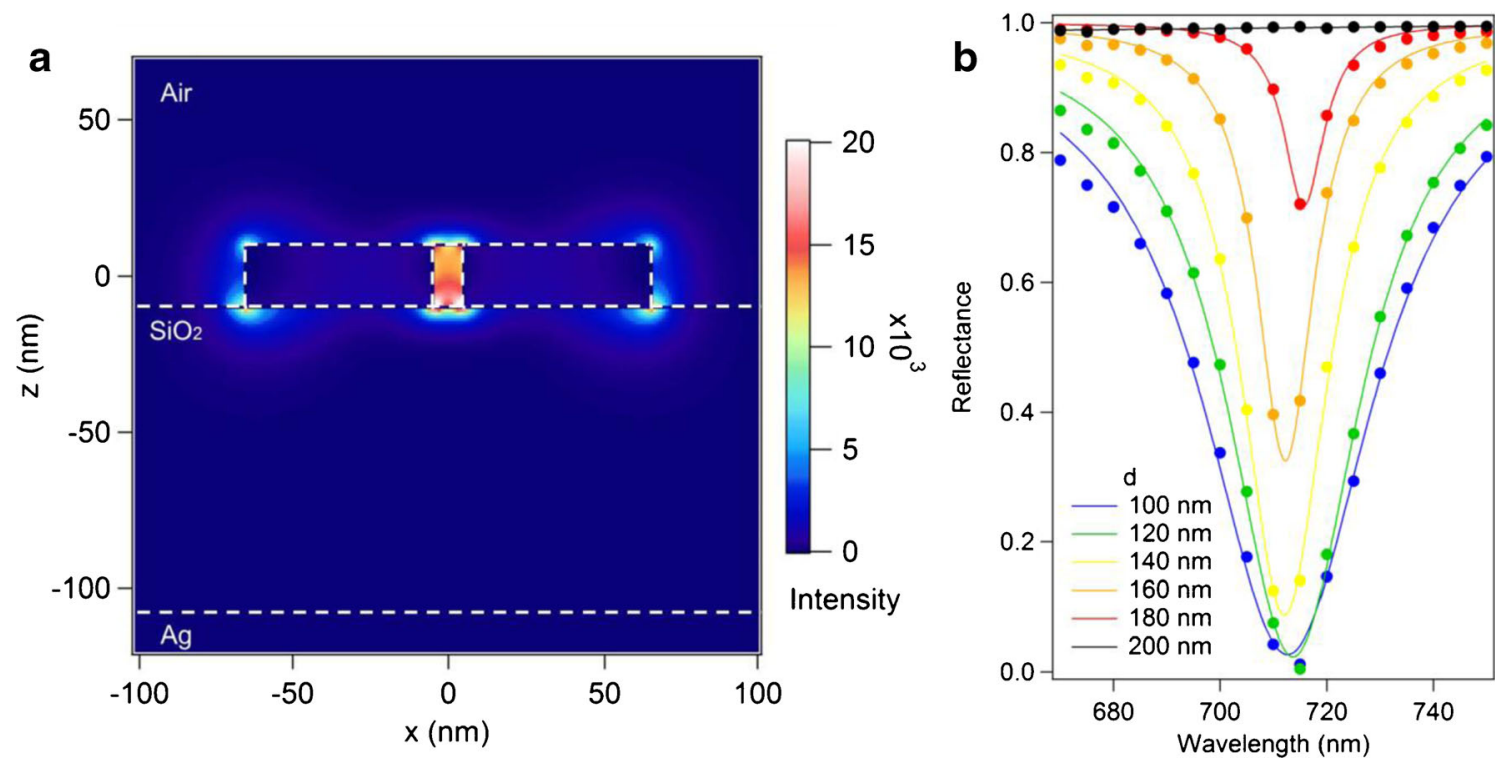

Fig. 3 a Near-field distribution of the intensity close to a silver nanoantenna loaded with polystyrene. The nanoantenna is part of an array with a period of $500 \mathrm{~nm}$. The incident wavelength is $\lambda=710 \mathrm{~nm}$. b Reflectance for a periodic array of silver nanoantennas loaded with polystyrene on a

influence of an increase of the load refractive index on the optical response of the nanoparticle array, computations have been performed considering different values of this refractive index for two different $\mathrm{SiO}_{2}$ layer thicknesses, namely $d=100$ and $180 \mathrm{~nm}$. Figure 4 shows the intensity enhancement factor $I / I_{0}$, where $I_{0}$ is the incident intensity and $I$ is the intensity at the nanogap center (Fig. 3a), and the reflectance spectra for different values of the refractive index of the material loaded in the gap. It is clearly observed that an increase of the refractive index leads to a redshift of the surface plasmon resonance supported by the nanoantennas.

The resonance wavelength $\lambda_{I}$ can be expressed as [33]:

$\lambda_{I}=\lambda_{c}+\alpha n_{I}$,

where $\lambda_{\mathrm{c}}$ is the dip wavelength computed considering only the linear refractive index of the polystyrene, $n_{I}$ is the variation of the refractive index, and $\alpha$ is a weighting coefficient, which can be extracted using a fitting procedure. The nonlinear Kerr effect in the polystyrene load is expected to have a similar detuning effect and to also modify the resonant wavelength of the nanoantenna array. Taking into account this effect, Eq. (4) can be rewritten as:

$\lambda_{I}=\lambda_{c}+\alpha n_{2} I$.

The data reported in Fig. 4 allow determining the relation between the reflectance and the enhancement factor for a given configuration at different wavelengths; a linear relation is observed between the enhancement factor and the reflectance. This allows us to deduct a
$\mathrm{SiO}_{2}-\mathrm{Ag}$ layered substrate as a function of the incident wavelength for different values of the $\mathrm{SiO}_{2}$ layer thickness $d$. The continuous lines are fits using Eq. (2)

relation between the reflected intensity $I_{\text {ref }}$ and the intensity in the gap:

$I=\beta I_{\text {ref }}+\gamma$.

Finally, the reflected intensity becomes:

$I_{\text {ref }}=I_{0} \frac{4\left(\lambda-\lambda_{I}\right)^{2}+w^{2}-\frac{2 A_{0} w}{\pi}}{4\left(\lambda-\lambda_{I}\right)^{2}+w^{2}}$,

with $\lambda_{I}=\lambda_{c}+\alpha n_{2}\left(\beta I_{\text {ref }}+\gamma\right)$. Equation (7) is a third order algebraic equation that provides the reflectance as a function of the incident intensity. All the parameters involved in this relation are functions of the dielectric spacer thickness $d$. In the following, we use the relation (7) to determine the influence of the reflecting silver surface and the $\mathrm{SiO}_{2}$ layer on the nonlinear response of the nanoantenna array.

Figure 5 shows the reflectance as a function of the incident intensity for different incident wavelengths and $\mathrm{SiO}_{2}$ layer thicknesses $d$. The first considered wavelength is $\lambda=710 \mathrm{~nm}$ corresponding to the resonant wavelength of the surface plasmon mode supported by the nanoantennas (see Fig. 1). For the two considered thicknesses, a modulation of the reflectance is observed, but the system does not support optical bistability (Fig. 5a). Indeed, it was demonstrated in the case of an isolated nanoantenna that optical bistability can be observed only if the relation

$\lambda>\lambda_{c}+\frac{\sqrt{3} w}{2}$ 

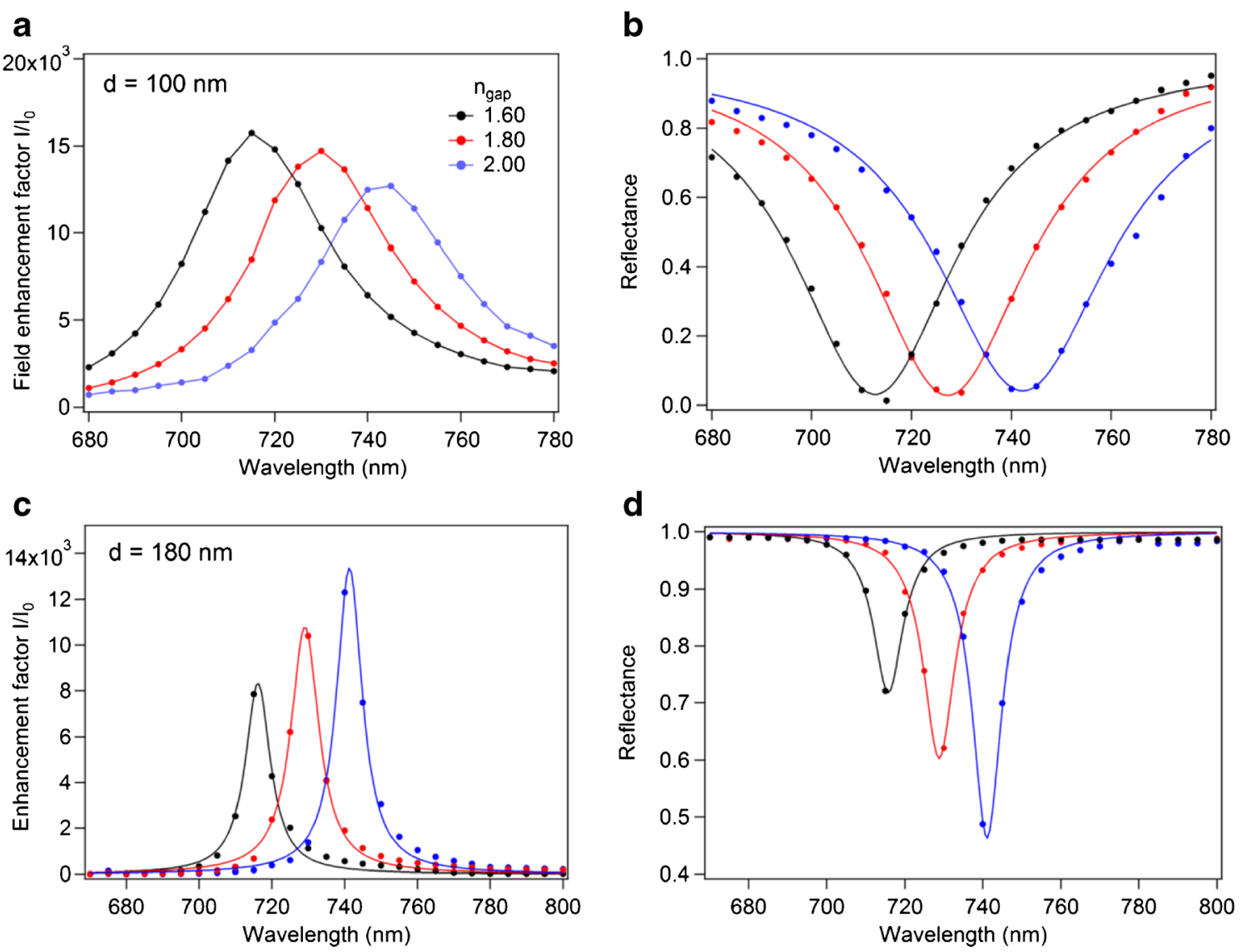

Fig. 4 a, $\mathbf{c}$ Enhancement factor $I / I_{0}$ evaluated at the nanoantenna gap center and $\mathbf{b}$, $\mathbf{d}$ reflectance computed for different values of the optical refractive index of the loaded material $n$ as a function of the illumination wavelength. Two $\mathrm{SiO} 2$ layered thicknesses are considered: $\mathbf{a}, \mathbf{b} d=100 \mathrm{~nm}$ or $\mathbf{c}, \mathbf{d} d=180 \mathrm{~nm}$

is satisfied [33]. This is clearly not the case here. Eq. (8) indicates that a longer wavelength is required for the observation of optical bistability. Figure $5 \mathrm{~b}$ shows the reflectance as a function of the incident intensity for an incident wavelength $(\lambda=730 \mathrm{~nm})$ slightly longer than the linear resonant wavelength. Even though only a modulation of the reflectance is observed for $d=100 \mathrm{~nm}$, optical bistability is clearly visible for $d=180 \mathrm{~nm}$. Note that the unstable branch is not shown in Fig. 5. The different behaviors are explained by the modification of the resonance width. The presence of the reflecting surface modifies the radiative properties of the nanoantenna array and the quality factor of the surface plasmon resonance (see Fig. 4) [18]. The resonance is narrower for $d=180 \mathrm{~nm}$, and optical bistability is observed in agreement with Eq. (8). Finally, an even longer wavelength is considered $(\lambda=770 \mathrm{~nm})$. Figure $5 \mathrm{c}$ shows that at this wavelength, optical bistability is observed for the two considered

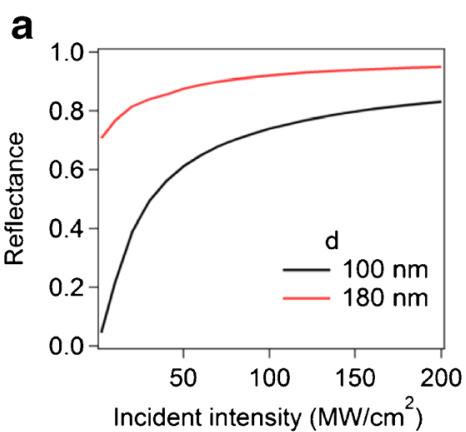

Fig. 5 Reflectance as a function of the incident intensity evaluated for a $\mathrm{SiO} 2$ thickness $d=100 \mathrm{~nm}$ (black curves) and $d=180 \mathrm{~nm}$ (red curves) considering the influence of the polystyrene Kerr effect on the
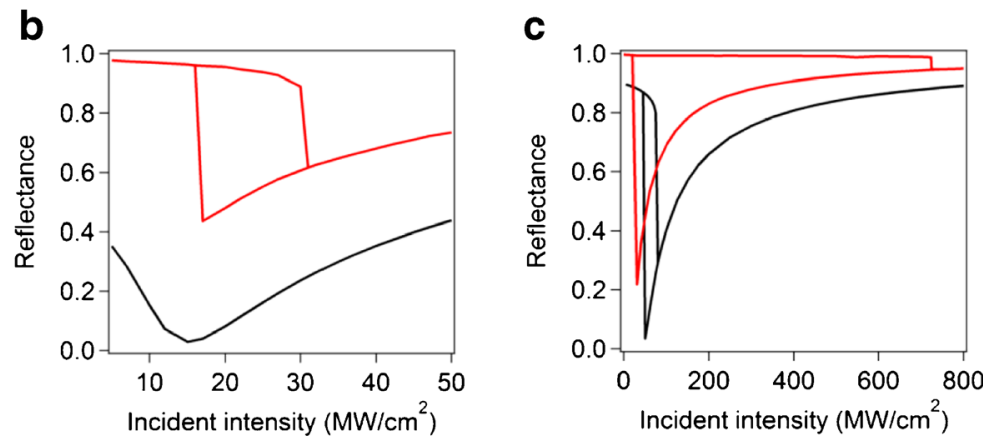

nanoantenna array response, as discussed in the text. The incident wavelength is $\mathbf{a} \lambda=710 \mathrm{~nm}, \mathbf{b} \lambda=730$ and $\lambda=770 \mathrm{~nm}$ 
thicknesses $d$. Interestingly, important parameters such as the intensity threshold and the area of the hysteresis cycle depend on the distance between the nanoantenna array and the reflecting surface. Despite the simplicity of the proposed approach, a clear modification of the intensity threshold is observed. Indeed, Fig. $5 \mathrm{c}$ shows that a small variation $(80 \mathrm{~nm})$ of the distance between the silver film and the nanoantenna array modifies the intensity threshold by $\sim 10$. Note that the intensity threshold depends on both the resonance width $w$ and the field enhancement in the nanogap (see Fig. 4). Furthermore, it is worth noting that optical bistability can be achieved at lower incident intensities than those reported for isolated nanoantennas [33]. These results clearly demonstrate that a reflecting surface can be used to control the nonlinear response of plasmonic nanoantenna array. Nanoantenna arrays loaded with nonlinear materials were proposed as optical memories, transistors, and switches at the nanoscale $[36,37]$. The results presented in this article show that a reflecting surface is a convenient tool for tailoring their optical properties and controlling their operation. From an experimental point of view, the addition of a polystyrene load in the nanoantenna gaps can be very challenging. One possibility is then to completely cover the nanoantenna array with a polystyrene layer. In this configuration, the Kerr effect is significant only in the nanoantenna gap, where the electromagnetic is enhanced by several orders of magnitude.

\section{Conclusions}

In summary, the influence of a reflecting surface on the nonlinear response of a nanoantenna array was investigated using a surface integral equation method and an analytical model for describing the Kerr effect in nanoantennas loaded with a nonlinear material. The influence of a silver layer on the nonlinear response of the nanoantenna array was investigated in details. Interestingly, it was shown that different behaviors are observed when the distance between the nanoantenna array and the silver layer is modified. This feature can be used to control the nonlinear response of metamaterials combining plasmonic and Kerr materials. The strength of the proposed approach is its simplicity and the possibility to extend it to any plasmonic system, such as the nonlinear nanoshells (nonlinear nanocores covered with silver shells) introduced by Argyropoulos et al. [37] or the hybrid metal-dielectric nanoantennas (which are composed of crystalline silicon and silver nanoparticles) proposed by Noskov et al. [36] for example. Furthermore, the method proposed in this article can be combined with other ones in order to achieve efficient optical nanomemories or nanoswitches. For example, it was reported that including a gain medium, which can be supplied by molecules or quantum dots, boosts the performance of plasmonic devices [45-47].

Acknowledgments This work was supported by the Swiss National Science Foundation (grants 200020_153662 and 406440_131280).

\section{References}

1. Maier SA (2007) Plasmonics: fundamentals and applications. Springer

2. Kottmann JP, Martin OJF (2001) Plasmon resonant coupling in metallic nanowires. Opt Express 8:655-663

3. Prodan E, Radloff C, Halas NJ, Nordlander P (2003) A hybridization model for the plasmon response of complex nanostructures. Science 302:419-422

4. Halas NJ, Lal S, Chanq WS, Link S, Nordlander P (2011) Plasmons in strongly coupled metallic nanostructures. Chem Rev 111:39133961

5. Mühlschlegel P, Eisler HJ, Martin OJF, Hecht B, Pohl DW (2005) Resonant optical antennas. Science 308:1607-1608

6. Novotny L, van Hulst NF (2011) Antennas for light. Nat Photonics 5: 83-90

7. Biagioni P, Huang JS, Hecht B (2012) Nanoantennas for visible and infrared radiation. Rep Prog Phys 75:024402

8. Fischer H, Martin OJF (2008) Engineering the optical response of plasmonic nanoantennas. Opt Express 16:9144-9154

9. Farahani JN, Pohl DW, Eisler HJ, Hecht B (2005) Single quantum dot coupled to a scanning optical antenna: a tunable superemitter. Phys Rev Lett 95:017402

10. Curto AG, Volpe G, Taminiau TH, Kreuzer MP, Quidant R, van Hulst NF (2010) Unidirectional emission of a quantum dot coupled to a nanoantenna. Science 329:930-933

11. Taminiau TH, Stefani FD, van Hulst NF (2011) Optical nanorod antennas modeled as cavities for dipolar emitters: evolution of suband super-radiant modes. Nano Lett 11:1020-1024

12. Ward DR, Grady NK, Levin CS, Halas NJ, Wu Y, Nordlander P, Natelson D (2007) Electromigrated nanoscale gaps for surfaceenhanced Raman spectroscopy. Nano Lett 7:1396-1400

13. Zhang W, Fischer H, Schmid T, Zenobi R, Martin OJF (2009) Modeselective surface-enhanced Raman spectroscopy using nanofabricated plasmonic dipole antennas. J Phys Chem C 113: $14672-14675$

14. Huang L, Maerkl SF, Martin OJF (2009) Integration of plasmonic trapping in a microfluidic environment. Opt Express 17:6018-6024

15. Zhang W, Huang L, Santschi C, Martin OJF (2010) Trapping and sensing $10 \mathrm{~nm}$ metal nanoparticles using plasmonic dipole antennas. Nano Lett 10:1006-1011

16. Juan ML, Righini M, Quidant R (2011) Plasmon nano-optical tweezers. Nat Photonics 5:349-356

17. Lévêque G, Martin OJF (2006) Optical interactions in a plasmonic particle coupled to a metallic film. Opt Express 14:9971-9981

18. Seok TJ, Jamshidi A, Kim M, Dhuey S, Lakhani A, Choo H, Schuck PJ, Cabrini S, Schwartzberg AM, Bokor J, Yablonovitch E, Wu MC (2011) Radiation engineering of optical antennas for maximum field enhancement. Nano Lett 11:2606-2610

19. Min Q, Pang Y, Collins DJ, Kuklev NA, Gottselig K, Steuerman DW, Gordon R (2011) Substrate-based platform for boosting the surfaceenhanced Raman of plasmonic nanoparticles. Opt Express 19:16481655

20. Fernandez-Garcia R, Rahmani M, Hong M, Maier SA, Sonnefraud Y (2012) Use of a gold reflecting-layer in optical antenna substrates for 
increase of photoluminescence enhancement. Opt Express 21: $12552-12561$

21. Kauranen M, Zayats AV (2012) Nonlinear plasmonics. Nat Photonics 6:737-748

22. Biagioni P, Brida D, Huang JS, Kern J, Duo L, Hecht B, Finazzi M, Cerullo G (2012) Dynamics of four-photon photoluminescence in gold nanoantennas. Nano Lett 12:2941-2947

23. Berthelot J, Bachelier G, Song M, Rai P, Francs G, Dereux A, Bouhelier A (2012) Silencing and enhancement of second harmonic generation in optical gap antennas. Opt Express 20:10498-10508

24. Slablab A, Le Xuan L, Zielinski M, de Wilde Y, Jacques V, Chauvat D, Roch JF (2012) Second-harmonic generation from coupled plasmon modes in a single dimer of gold nanospheres. Opt Express 20: 220-227

25. Butet J, Thyagarajan K, Martin OJF (2013) Ultrasensitive optical shape characterization of gold nanoantennas using second harmonic generation. Nano Lett 13:1787-1792

26. Thyagarajan K, Butet J, Martin OJF (2013) Augmenting second harmonic generation using Fano resonances in plasmonic systems. Nano Lett 13:1847-1851

27. Metzger B, Hentschel M, Lippitz M, Giessen H (2013) Thirdharmonic spectroscopy and modeling of the nonlinear response of plasmonic nanoantennas. Opt Lett 37:4741-4743

28. Navarro-Cia M, Maier SA (2012) Broad-band near-infrared plasmonic nanoantennas for higher harmonic generation. ACS Nano 6: $3537-3544$

29. Danckwerts M, Novotny L (2007) Optical frequency mixing at coupled gold nanoparticles. Phys Rev Lett 98:026104

30. Wurtz GA, Pollard R, Zayats AV (2006) Optical bistability in nonlinear surface-plasmon polaritonic crystals. Phys Rev Lett 97:057402

31. Shen Y, Wang GP (2008) Optical bistability in metal gap waveguide nanocavities. Opt Express 16:8421-8426

32. Chen PY, Alù A (2010) Optical nanoantenna arrays loaded with nonlinear materials. Phys Rev B 82:235405

33. Zhou F, Liu Y, Li ZY, Xia Y (2010) Analytical model for optical bistability in nonlinear metal-antennae involving Kerr materials. Opt Express 18:13337-13344
34. Chen PY, Argyropoulos C, Alù A (2012) Enhanced nonlinearities using plasmonic nanoantennas. Nanophotonics 1:221-233

35. Argyropoulos C, Ciracì C, Smith DR (2014) Enhanced optical bistability with film-coupled plasmonic nanocubes. App Phys Lett 104:063108

36. Noskov RE, Krasnok AE, Kivshar YS (2012) Nonlinear metaldielectric nanoantennas for light switching and routing. New J Phys 14:093005

37. Argyropoulos C, Chen PY, Monticone F, D'Aguanno G, Alù A (2012) Nonlinear plasmonic cloaks to realize giant all-optical scattering switching. Phys Rev Lett 108:263905

38. Gallinet B, Kern AM, Martin OJF (2010) Accurate and versatile modeling of electromagnetic scattering on periodic nanostructures with a surface integral approach. J Opt Soc Am A 27:2261-2271

39. Gallinet B, Martin OJF (2010) Scattering on plasmonic nanostructures arrays modeled with a surface integral formulation. Photonics Nanostruct Fund App 8:278-284

40. Kern AM, Martin OJF (2009) Surface integral formulation for 3D simulation of plasmonic and high permittivity nanostructures. J Opt Soc Am A 26:732-740

41. Johnson PB, Christy RW (1972) Optical constants of the noble metals. Phys Rev B 6:4370-4379

42. Ameling R, Giessen H (2013) Microcavity plasmonics: strong coupling of photonic cavities and plasmons. Laser Phot Rev 7:141-169

43. Boyd RW (1992) Nonlinear optics. Academic Press, New York

44. Liu Y, Qin F, Zhou F, Li ZY (2009) Ultrafast and low-power photonic crystal all-optical switching with resonant cavities. J App Phys 106: 083102

45. Li ZY, Xia YN (2010) Metal nanoparticles with gain toward singlemolecule detection by surface-enhanced Raman scattering. Nano Lett 10:243-249

46. Khurgin JB, Sun G (2012) Practicality of compensating the loss in the plasmonic waveguides using semiconductor gain medium. Appl Phys Lett 100:011105

47. Khurgin JB, Sun G (2013) Plasmonic enhancement of the third order nonlinear optical phenomena: figures of merit. Opt Express 21:27460-27480 